Relations industrielles

Industrial Relations

\title{
Les ententes individuelles et la convention collective
}

\section{Claude D'Aoust}

Volume 38, numéro 1, 1983

URI : https://id.erudit.org/iderudit/029333ar

DOI : https://doi.org/10.7202/029333ar

Aller au sommaire du numéro

Éditeur(s)

Département des relations industrielles de l'Université Laval

ISSN

0034-379X (imprimé)

1703-8138 (numérique)

Découvrir la revue

Citer cet article

D’Aoust, C. (1983). Les ententes individuelles et la convention collective.

Relations industrielles / Industrial Relations, 38(1), 155-158.

https://doi.org/10.7202/029333ar

Tous droits réservés @ C Département des relations industrielles de l'Universite Laval, 1983
Ce document est protégé par la loi sur le droit d'auteur. L’utilisation des services d'Érudit (y compris la reproduction) est assujettie à sa politique d'utilisation que vous pouvez consulter en ligne.

https://apropos.erudit.org/fr/usagers/politique-dutilisation/ 


\section{DROIT DU TRAVAIL}

\section{Les ententes individuelles et la convention collective}

\section{Claude D'Aoust}

La relation entre le contrat individuel de travail et la convention collective a donné naissance à deux écoles de pensée, l'une tenant pour absolues les normes conventionnelles et l'autre les tenant pour relatives. Entendons par là que, selon cette dernière conception, les conditions de travail négociées seraient «minimales» et il serait ainsi, par contrat individuel de travail, permis au salarié de stipuler des conditions meilleures; dans la première optique, la convention collective occupe toute la place. Cette question a été amplement discutée chez nous ${ }^{1}$ et je voudrais l'aborder maintenant sous un angle différent. Comme quoi la question sous étude n'est pas près d'être réglée.

Une décision albertaine en est l'occasion ${ }^{2}$. D'entrée de jeu, notons qu'un article du Code scolaire albertain permet expressément la détermination partielle des conditions de travail par entente individuelle ${ }^{3}$. Cela étant acquis, il reste à déterminer l'interaction du contrat individuel et de la con-

* Claude D’AOUST, professeur, École des Relations Industrielles, Université de Montréal, Montréal, P.Q.

1 Voir par exemple: Pierre VERGE, «Le problème des ententes individuelles plus favorables que la convention collective», Relations industrielles, vol. 22, no 2, 1967, p. 281; Pierre VERGE, «L'embauchage d'un salarié lié par une convention collective», Relations industrielles, vol. 26, no 2, 1971, p. 502; Claude D'AOUST, «Les ententes individuelles parallèles et la convention collective: note sur une décision récente», Relations industrielles, vol. 33, no 3, 1978, p. 564.

2 Corry v. Board of Trustees of Calgary School District No. 19, Alberta Court of Queen's Bench, May 21, 1982, 82 CLLC parag. 14195.

3 Voir: The School Act, R.S.A. 1970 c. 329, article 74, parag. 3:

$74(\ldots)$

(3) Subject to subsection (2) but notwithstanding any other agreement to the contrary the terms and conditions of a contract of employment between a board and a teacher shall be

(a) except in the case of a teacher excluded under section 82, subsection (5), the terms and conditions negotiated under The Alberta Labour Act and agreed between the board and an organization representing teachers,

(b) sections 74 to 81 of this Act, and

(c) the terms and conditions agreed between the board and the teacher,

and any contract excluding or purporting to exclude the provisions of clauses (a) and (b) is void.

Une erreur de rédaction ou de transcription semble s'être glissée dans la loi, vérifiée au texte; le sous-paragraphe b) devrait se lire: the terms and conditions provided for by sections 74 to 81 of this Act...

Relat. ind., vol. 38, no 1, 1983 @ PUL ISSN 0034-379 X 
vention collective. Il ressort de l'article 74, paragraphe 3, du School Act que les conditions de travail définies par les conventions collectives - de même que celles établies par la loi même - ne peuvent être écartées par entente individuelle; l'intérêt de la décision albertaine réside donc ailleurs. Examinons d'abord les faits.

Le 7 mars 1979, le demandeur recevait de son employeur une réponse favorable à sa demande de congé de perfectionnement avec solde partielle. Sous pli était inclus un formulaire d'acceptation pourvu d'espaces blancs pour l'inscription de la date et la signature. Cette lettre-formulaire renvoyait à deux documents émanant de l'employeur et comportait l'acceptation des conditions y contenues ${ }^{4}$.

Le congé d'études allait du $1^{\mathrm{er}}$ septembre 1979 à la fin août 1980. Corry suivit une première tranche de cours de septembre à avril, et il était inscrit à un autre cours s'étendant du 14 mai au 31 juin 1980 .

Survinrent alors deux événements.

D'abord, l'employeur lui demande d'assurer une charge d'enseignement à temps plein, du 8 mai au 28 juin 1980. Il fut convenu qu'il recevrait alors son plein salaire en sus de la rémunération tenant lieu de salaire attaché à son statut d'enseignant en congé d'études. De fait, il assuma sa pleine charge de travail en même temps qu'il suivait les cours prévus durant la période allant du 14 au 27 mai 1980.

Mais le 27 mai, le syndicat déclencha une grève légale qui se poursuivit jusqu'au 25 septembre 1980. Corry emboîta le pas. Il cessa d'exercer ses fonctions d'enseignements à temps partiel d'une part et ne réintégra son poste régulier, une fois son congé terminé, qu'à la fin de la grève. Cependant, du 27 mai au 30 juin, il continua et compléta avec succès le cours de perfectionnement qu'il avait entrepris.

Du point de vue juridique, certains aspects de la décision présentent peu ou pas d'intérêt. Ainsi, vu l'article 74 du School Act, il est clair qu'un contrat individuel de travail peut validement être conclu concuremment avec une convention collective. Cette disposition fait du secteur de l'éducation un cas particulier et ainsi la question générale du statut du contrat de travail en régime de négociation collective se trouve éludé.

Le contrat individuel de travail peut contenir des clauses sur des sujets autres que ceux prévus à la convention ou par la loi. Mais il est à noter en l'espèce que le contrat renvoyait à la convention collective en ce sens que les documents mentionnés dans la lettre d'acceptation, et traitant du système de congé de perfectionnement, reprenaient en substance certaines dispositions de la convention collective. En ce sens, une analogie peut être faite avec le renvoi législatif, technique par laquelle une législation est en quelque

4 Le texte se lit, en partie: “... I have read the Summary of Regulations governing Professional Improvement Leaves, the School Board Regulation no 2,030.5 and the procedures outlined in your letter of March 7th 1979, and I am prepared to accept the conditions outlined in these documents..." 
sorte incorporée à une autre 5 . Or, qu'advient-il lorsque la loi faisant l'objet du renvoi (i.e. la loi incorporée) est abrogée? Ce n'est pas le lieu de répondre à cette question ${ }^{6}$; mais poursuivons l'analogie et nous verrons en quels termes elle s'est ici posée.

L'article 11 de la convention collective contenait des dispositions «essentielles» et des dispositions "procédurales» relatives au congé de perfectionnement ${ }^{7}$. Ces conditions - et d'autres - étaient reprises dans un des documents mentionnés dans la lettre d'acceptation. C'est ainsi qu'elles ont survécu à l'expiration de la convention collective.

Que penser de cette solution? L'on sait que les conditions de travail prévues dans la convention collective sont impératives, en vertu de l'article $67,1 \mathrm{er}$ alinéa de notre Code du travail. Rien donc, à première vue, n'interdit de les reproduire dans un contrat individuel de travail ${ }^{8}$. Le juge Judson, dans le célèbre arrêt Paquet ne s'exprimait pas autrement ${ }^{9}$. Qu'on l'ait fait en l'espèce par l'intermédiaire d'un règlement patronal ne me paraît pas devoir être retenu comme élément significatif sur lequel établir une distinction.

Mais l'article 59 C.t. ne s'oppose-t-il pas à ce qu'on prolonge la vie de la convention collective par entente individuelle? Posée en ces termes, la question ne peut que recevoir une réponse affirmative. En l'espèce, il ne s'agissait pas de faire survivre la convention collective au-delà de son terme, produisant ainsi par la volonté d'un seul des effets juridiques à l'égard de tous les membres de l'unité d'accréditation; il s'agissait seulement d'en prolonger certains effets au bénéfice du seul signataire de l'entente.

On doit d'ailleurs signaler que cette affaire n'était qu'un cas particulier du problème plus général du versement du salaire ou de son équivalent en période de grève. Il est acquis qu'en ce cas les obligations principales de l'employeur et du salarié sont suspendues. Pas de travail, pas de salaire. Fort bien, sauf que ce principe n'est pas si absolu qu'on peut le penser à première vue. Le salarié qui est en congé-maladie continue de recevoir ses

5 Sur cette technique, son utilité et les problèmes qu'elle pose, consulter: Pierre-André CÔTÉ, Interprétation des lois, Les éditions Yvon Blais, Cowansville, 1982, p. 59 et seq.

À titre d'exemple voir la Loi sur les normes du travail, L.Q. 1979, c. 45, article 123.

6 Voir cependant COTÉ, ibid., pp. 60-61; Louis-Philippe PIGEON, Rédaction et interprétation des lois, $2^{\mathrm{e}}$ édition, Collection Études juridiques, Éditeur officiel du Québec, 1978, p. 65. Mentionnons simplement que la solution apportée au litige en l'espèce («renvoi contractuel», pourrions-nous dire) s'écarte de la règle applicable en matière de renvoi législatif.

7 Le terme "essentielles» est emprunté à PIGEON, ibid, p. 65; il correspond aux substantive rights par opposition aux procedural matters. La Cour, quant à elle, (loc. cit., p. 12980 ) oppose les clauses traitant des administrative matters à celle créant des substantive rights and obligations. Par exemple, les premières régissent le mode de sélection des candidats tandis que les secondes établissent la rémunération tenant lieu de salaire à laquelle auront droit les candidats choisis.

8 On peut d'ailleurs en dire autant du règlement intérieur, relativement aux clauses susceptibles d'y être incorporées.

9 Le Syndicat catholique des employés de magasins de Québec Inc. c. Paquet, |1959| R.C.S. 206, pp. 212-13. 
prestations d'assurance-salaire si une grève est déclenchée ou un lock-out décrété durant son absence. Mais cela ne peut se faire qu'en vertu d'une disposition de la convention collective expirée ou d'une clause du contrat d'assurance-salaire souscrit auprès d'un tiers-assureur. Cela illustre, sans le démontrer, qu'il est juridiquement possible de pourvoir au versernent de substituts de salaire au-delà de l'échéance de la convention collective.

Dans l'adage "pas de travail, pas de salaire», il convient donc de définir exactement le terme «salaire». Il en va de même du terme «travail»; il ne peut être pris au sens strict que si le terme «salaire» est également interprété strictement et vice-versa. En l'occurrence, les sommes étaient versées en contrepartie des cours universitaires suivis et réussis par l'enseignant et de l'obligation de demeurer à l'emploi de la commission scolaire un certain nombre d'années après la fin de son congé. On voit par là qu'il y a péril en la demeure lorsqu'on tente de solutionner des questions complexes au moyen de maximes simplistes ${ }^{10}$.

En guise de conclusion, cette décision albertaine vaut d'être signalée pour les réflexions qu'elle est susceptible de nous inspirer sur la délicate question du rapport entre l'entente individuelle et la convention collective. Elle montre que la problématique ne saurait être réduite à la question de savoir si les normes de la convention sont minimales ou impératives. Il y a en effet des cas où il importe davantage de dégager l'effet complémentaire des deux actes juridiques quand tous deux traitent d'un sujet plutôt que de déterminer lequel l'emporte sur l'autre.

10 Il convient de rendre compte d'un dernier argument, secondaire pour ne pas dire oiseux, soulevé par l'employeur. Il prétendait qu'ayant accepté un emploi temporaire à temps plein, en violation d'une règle a l'encontre, Corry avait renoncé à ses prestations de congé. Une autre règle prévoyant une exception, à condition d'obtenir la permission d'un haut fonctionnaire du réseau scolaire ("prior approval by the Chief Superintendent of Schools"), la Cour conclut que cette exception s'étendait implicitement à un emploi au service de la commission scolaire elle-même. 